University of Nebraska - Lincoln

DigitalCommons@University of Nebraska - Lincoln

Sociology Department, Faculty Publications

Sociology, Department of

$4-2007$

\title{
Early-Onset Alcohol Use Among Native American Youth: Examining Female Caretaker Influence
}

\author{
Melissa L. Walls \\ University of Minnesota-Duluth, mlwalls@umn.edu \\ Les B. Whitbeck \\ University of Nebraska-Lincoln, Iwhitbeck2@unl.edu \\ Dan R. Hoyt \\ University of Nebraska-Lincoln, dhoyt2@unl.edu \\ Kurt D. Johnson \\ Pennsylvania State University, kdj11@psu.edu
}

Follow this and additional works at: https://digitalcommons.unl.edu/sociologyfacpub

Walls, Melissa L.; Whitbeck, Les B.; Hoyt, Dan R.; and Johnson, Kurt D., "Early-Onset Alcohol Use Among Native American Youth: Examining Female Caretaker Influence" (2007). Sociology Department, Faculty Publications. 206.

https://digitalcommons.unl.edu/sociologyfacpub/206

This Article is brought to you for free and open access by the Sociology, Department of at DigitalCommons@University of Nebraska - Lincoln. It has been accepted for inclusion in Sociology Department, Faculty Publications by an authorized administrator of DigitalCommons@University of Nebraska - Lincoln. 
Published in Journal of Marriage and Family 69:2 (May 2007), pp. 451-464; doi: 10.1111/j.1741-3737.2007.00376.x Copyright @ 2007 National Council on Family Relations; published by John Wiley \& Sons. Used by permission.

Published online April 8, 2007.

\title{
Early-Onset Alcohol Use Among Native American Youth: Examining Female Caretaker Influence
}

\author{
Melissa L. Walls, Department of Sociology/Anthropology, University of Minnesota-Duluth \\ Les B. Whitbeck, Department of Sociology, University of Nebraska-Lincoln \\ Dan R. Hoyt, Department of Sociology, University of Nebraska-Lincoln \\ Kurt D. Johnson, Social Science Research Institute at the Pennsylvania State University \\ Corresponding author - M. L. Walls, mlwalls@d.umn.edu
}

\begin{abstract}
This article investigates the influence of female caretaker substance use on early-onset youth drinking among Native American families in the Northern Midwest. Data include 603 Native American families, with reports from female caretakers and youths aged 10-13 years. Two potential caretaker influences are taken into account: adolescent modeling of caretaker behaviors and the effects of caretaker substance abuse on parenting. Results of bivariate and path analysis provide support for the influence of caretaker substance use on adolescent drinking from both perspectives; these effects vary, however, depending on the type or degree of adult substance use, or both.
\end{abstract}

Keywords: adolescent substance use, Native Americans, parenting, parenthood

A lcohol use, abuse, treatment, and prevention are all major issues of public concern in the United States today. Problems surrounding alcohol use and abuse have serious economic, psychological, physiological, and social consequences (Rivers, 1994). According to the 2002 National Survey on Drug Use and Health, $12 \%$ of U.S. 12-year- olds report lifetime alcohol use, with rates increasing throughout adolescence until a peak of $91 \%$ at age 21 (Substance Abuse and Mental Health Services Administration, 2002). Although such alcohol experimentation throughout the adolescent years is generally a normative behavior, early onset of alcohol use is associated with a variety of developmental problem outcomes (Kaplow et al., 2002). For example, earlier age of onset (before 15 years of age) has been found to be associated with lower achievement, academic problems, delinquent and antisocial behavior, later drug abuse and alcoholism, and adulthood problems with employment and criminal and violent behavior (Ellickson, Tucker, \& Klein, 2003; Kandel, Yamaguchi, \& Chen, 1992; McGue, Iacono, Legrand, Malone, \& Elkins, 2001). In addition, such early substance use may play a role in three of the five leading causes of injury death for adolescents aged 10-14 years: motor vehicle accidents, suicide, and homicide (Centers for Disease Control and Prevention, 2001). Because of these detrimental effects associated with earlier drinking initiation, it is important for researchers to determine factors that may be predictive of early onset use (Kaplow et al., 2002). 
Patterns and prevalence of alcohol use vary according to demographic traits. Although a variety of trends may be explored across gender, age, and ethnic groups, central to the importance of this study are the alcohol-related characteristics and behaviors of Native American adolescents. United States, Canadian national, and multisite estimates of substance use rates find that on average, native youths show higher rates of drinking and drug use than most other racial or ethnic groups (Bachman et al., 1991; Beauvais, 1996; Gfellner, 1994; Plunkett, 2000). Native youth may be at particularly high risk in terms of early onset of substance use (May, 1982, 1986) and disproportionately high rates of nonexperimental substance use (see Herring, 1994). Alcoholism death rates among young Native Americans (aged 15-24) has been estimated at 3.4 deaths per 100,000 compared to a rate of only 0.3 for the overall U.S. population (Indian Health Service, 1998-1999). Problems linked to alcohol use may be heightened for youth residing on reservations, who have been found to be more likely than nonreservation dwelling Native Americans and European Americans to report traffic tickets, car accidents, arrests, money problems, school troubles, fights, and property damage resulting from alcohol use (with White youth showing the lowest rates of such problems). These issues exclude potential damages to physical, emotional, social, and spiritual well-being (Beauvais, 1992). Thus, Native American youth in general are an especially high-risk group in terms of early exposure, prevalence of use, and problems associated with alcohol.

Despite these general findings, measurements of alcohol prevalence are complicated when one considers that the two million Native American and Alaskan Natives in the Unites States fall into over 300 different tribal or language groups, with variability in histories, levels of enculturation, and traditional practices both among individual tribes and within the smaller communities that make up these tribes (Beauvais, 1998). Furthermore, many Native people affiliate themselves with more than one specific tribal group, and others may identify as multiethnic. These patterns make the validity of generalizing "overall" drinking patterns to specific Native American groups questionable, calling forth the need to assess alcohol-related characteristics (i.e., prevalence, predictors, policy, and prevention focus) for different tribal groups individually (May, 1995).
The purpose of this article was to examine how various caretaker substance use characteristics and parenting processes influence the onset of youth alcohol use within a single Native American culture in the Northern Midwest. This research is significant in that it includes measures of parental influence that have received little attention in the current Native American-specific literature (but see Rodgers \& Fleming, 2003; Swaim, Oetting, Thurman, Beauvais, \& Edwards, 1993).

\section{Literature review}

Despite a large and growing body of literature surrounding alcoholism among Native Americans, most of the research on adolescent alcohol and substance use concentrates on the majority population. In the Native American-specific work that has been done, there is a major gap in the research surrounding alcohol use and Native families (Mail \& Heurtin-Roberts, 2002). As is elaborated below, non-Native American-based literature frequently cites a social perspective of alcohol use focusing on the relationship between parental and offspring substance use (Anderson \& Henry, 1994; Barnes \& Farrell, 1992; Chassin \& DeLucia, 1996).

\section{Caretaker alcohol use and early-onset adolescent drinking}

Past research on parent or child drinking has found support for the direct influence of parental alcohol use on adolescent drinking. For example, a study by Barnes, Farrell, and Cairns (1986) found that adolescent drinking patterns reflect the drinking patterns of their parents. Similarly, others have found significant relationships between parental and adult norms surrounding alcohol and earlier adolescent ages of onset for drinking (Akers et al., 1979; Sieving, Maruyama, Carolyn, Williams, \& Perry, 2000). Another study (Li, Pentz, \& Chih-Ping, 2002) shows that substance using parents and friends are associated with adolescent substance use risk and that nonusing parents can act as a buffer to such risk. Although Ary, Tildesley, Hops, and Andrews (1993) did not find support for parental modeling of alcohol use in terms of concurrent use by children, they did find an association related to change in later (1-year followup) use of alcohol by youth. These studies contribute to an explanation of observational learning in which children adopt or imitate the drinking behaviors of their parents. 
A social learning perspective seems especially fitting in reference to the Native American group comprising this sample when considering the traditional centrality of family and a strong cultural emphasis on respecting elders. As suggested by Johnson and Johnson (1999), the influence of parental models is heightened in cultures where special importance is placed on family relationships and parental authority. This culture's traditional family structure and emphasis on the influence caretakers have on their children is illustrated further with consideration of the importance of harmony as the interconnectedness of all living things. Therefore, we see an interdependence of family members as sources of support and learning. As Rivers (1994) explains, learning through modeling often occurs when we adopt the behaviors of those we admire, wish to be like, or seek praise from. Learned drinking behaviors allow us a distinct way to identify and imitate behaviors of people in our lives who do the same.

\section{Caretaker alcohol use and parenting behaviors}

A second major direction of this area of research tests the indirect effects of caretaker drinking on their children's use of alcohol. These studies focus on how alcohol and substance use negatively affects parenting behaviors in terms of lowered monitoring (Barnes \& Farrell, 1992; DiClemente et al., 2001) and inconsistent parenting (Windle, 1996). Such instances of inadequate parenting can, in turn, increase the onset and rate of youth substance use (Barnes, Reifman, Farrell, \& Dintcheff, 2000; Beck, Boyle, \& Boekeloo, 2003). These studies suggest an influence of parental alcohol use on youth drinking through less effective parenting behaviors.

Coercion theory (Reid, Patterson, \& Snyder, 2002), with roots in social learning theory, states that differences in antisocial behaviors across individuals result from a compilation of children's daily social interactions. Basically, coercive interactions take on a cyclical pattern that continues to build over time. Such patterns, if not interrupted, may become the basis for antisocial behavior. Empirical evidence on the basis of concepts from coercion theory suggests that inadequate discipline and low levels of parental monitoring represent major factors for both the emergence and the maintenance of youth antisocial behaviors (Reid et al., 2002).

Although coercion theory has been mainly used to predict antisocial behavior in general, it is also capable of explaining how coercive interactions affect the more specific outcome of early adolescent drinking. This concept has been tested via Problem-Behavior Theory, which was initially developed for a study of alcohol abuse and other problem behaviors in a triethnic community that included Native Americans (Jessor, 1987). Jessor explains, "as it turns out in a fairly large and robust set of studies, the pattern of interrelations (between alcohol use and problem behavior in general) is systematic enough to suggest that problem drinking is part of a syndrome of adolescent problem behavior" (1987, p. 336).

Past research calls for further studies that examine a combination of both direct and indirect influences of caretaker effects on child drinking (Wills \& Yaeger, 2003; Windle, 1996). Despite a significant body of research concerning the associations between caretaker and youth substance and alcohol use, much remains to be learned about the specific ways in which these transmissions occur (Jacob \& Johnson, 1999), especially among Native American populations. By looking at the processes of observational learning (i.e., social learning theory) and ineffective parenting skills together (coercion theory), we might better understand how they operate both as separate and interactive processes.

\section{Other factors related to youth alcohol use}

Beyond our major focus on caretaker behaviors as they relate to early-onset alcohol use among Native American adolescents, past research dictates our decision to include several control variables that have been shown to influence adolescent alcohol use. A robust finding in the literature on youth substance use is that rates of alcohol use increase across adolescence; this finding has been consistent across time and within a variety of racial or ethnic groups (Wallace et al., 2003). Past research has also indicated a negative association between socioeconomic status and adolescent substance use, but only in conditions of extreme poverty (Hawkins, Catalano, \& Miller, 1992). Male adolescents have historically reported higher rates of substance use than their female peers (Bachman et al., 1991), although evidence points to a narrowing or closing, or both, of this gender gap over time (Donnermeyer, 1992; Wallace et al., 2003). Studies also have documented the troubles faced by young parents in terms of inadequate education, financial instability, and family conflict as 


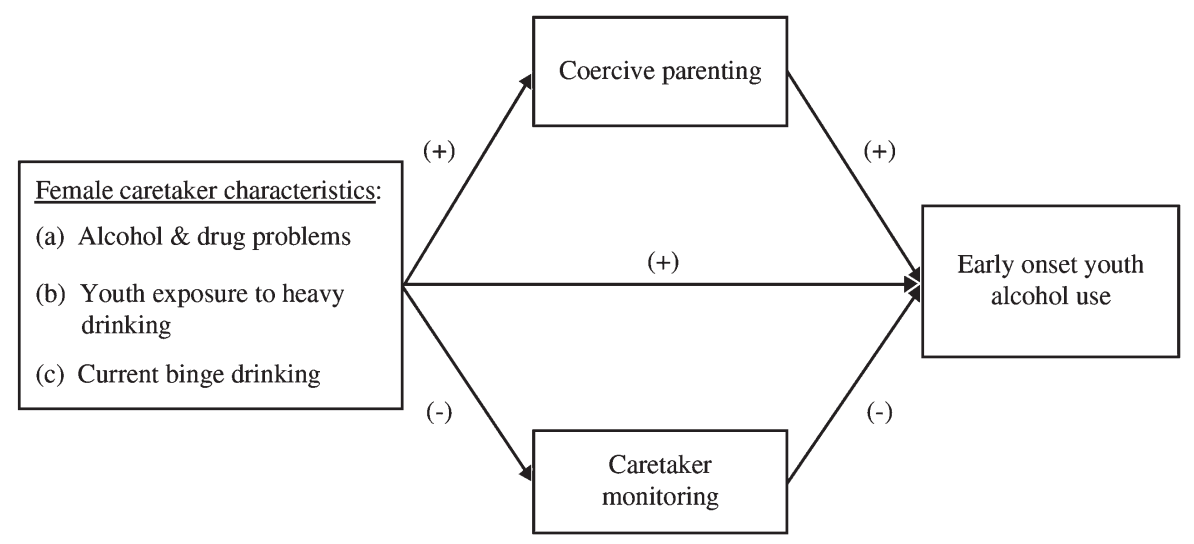

Figure 1. Conceptual Model

compared to older parents, and these age- and life course-specific stressors have been connected to child outcomes: More specifically, mother's age at delivery is negatively associated with youth outcomes, even into the child's adulthood (Hardy et al., 1997). On the basis of these studies, we control for youth age, household income, youth gender, and caretaker age in our analyses.

\section{Hypotheses (H1-H4)}

Figure 1 illustrates the conceptual model underlying the analyses for this study. First, on the basis of social learning theory, (H1) caretaker alcohol characteristics are expected to have a direct, positive association with adolescent alcohol use. As noted, prior research supports (H2) that caretaker alcohol characteristics will negatively affect parenting processes, here measured by higher levels of coercive parenting and lower levels of parental monitoring. In turn, and consistent with coercion theory, (H3) these less effective parenting processes will be associated with adolescent alcohol use. In terms of the control variables, $(\mathrm{H} 4)$ older youths, boys, those with lower household incomes, and youth living with younger female caretakers all will show a positive association with early-onset alcohol use.

\section{Method}

\section{Sample}

Data for this study were collected as part of a 3-year longitudinal study currently under way. The study was designed in partnership with 10 reservations and reserves and a university-based research team. As part of this partnership, the names of the reservations and reserves and potentially identifying cultural information are excluded from this article. On each reservation or reserve, Tribal Council-appointed advisory boards are responsible for handling personnel difficulties, advising the research team on questionnaire development, and reviewing or approving reports and proposals. All participating staff on the reservations and reserves (i.e., interviewers, site coordinators) are approved by advisory boards and are either enrolled tribal members or spouses of enrollees. Interviewers for this project were trained concerning methodological guidelines of personal interviewing.

Each participating tribe provided a list of families of tribally enrolled children aged 10-12 years who lived on or proximate to (within 50 miles) the reservation or reserve. We attempted to contact all families with a target child within the specified age range to achieve a population sample within participating communities of this cultural group. Families for this study were recruited through personal interviewer visits during which they were presented a traditional gift, an overview of the project, and an invitation to participate. Families were chosen for visits providing that at least one tribally enrolled child in the house was between the ages of 10 and 12 years (target adolescent). For those families who agreed to participate, both the target adolescent and at least one adult caretaker (and in some cases, two adults) were given $\$ 40$ upon completion of the interviews. In the rare case that more than one child in a household matched 
study selection criteria, each child was invited to participate and receive incentives. Responses from only one child per household (chosen by random selection), however, are included in our final data to avoid nested data issues. Overall response rate for Wave 1 was $79.4 \%$.

This article includes data from Year 1 of the study (subsequent waves of interviews are currently in progress). Of the 746 children and their caretakers interviewed, only cases that include interviews from female caretakers, most often biological mothers of target adolescents, are used for this study. The decision to focus on female caretakers was based on extreme heterogeneity in male caretaker relationships to the target adolescent (i.e., mother's boyfriend, uncle, grandfather, father), as well as the relatively few male compared to female adult respondents (227 men/686 women). In addition to this data reduction, we include only responses from U.S. residents in this study. Because of differences in legal systems, our Canadian Advisory Boards asked that the spanking question included in our measure of coercive parenting be excluded in the questionnaire administered at Canadian reserves.

The final sample is comprised of 603 adolescents and their adult female caretakers. From this group, caretakers ranged in age from 19 to 77 years. Most of the female adults were biological mothers $(80.3 \%)$ of the target adolescents. Of the remaining caretakers, $10.9 \%$ were the adolescent's grandmother, $2.3 \%$ stepmothers, $2.2 \%$ foster parents, $1.7 \%$ aunties, and the remaining $2.6 \%$ had some other relationship with the youth (i.e., sister, cousin, other relative, adoptive parent). Target adolescents were between 10 and 13 years of age (13-year-olds are those youth who experienced birthdays between recruitment and interview dates). Caretakers reported having an average of 4.4 children. Educational attainment among these female caretakers was distributed as follows: $13.5 \%$ reported less than a high school education; $37.7 \%$ said they had a high school diploma or General Equivalency Diploma; 41.6\% reported some college, technical, or vocational training; and the remaining $7.2 \%$ had college degrees or more. In terms of employment, 57.7\% of the caretakers reported having a full-time job, $10 \%$ had part-time employment, $17.9 \%$ said they were unemployed, and the remainder of adults said they were retired, students, disabled, or homemakers.

\section{Measures}

Because of the relative youth of this sample and our focus on the onset of alcohol use, we chose to use a measure of lifetime drinking prevalence rather than other more detailed measures of drinking frequency or severity, or both. Early-onset adolescent alcohol use is measured by three questions that ask target adolescents whether they had ever tried a drink (more than a sip) of (a) beer, (b) wine (not counting religious ceremonies), or (c) other alcoholic beverages. We created a dichotomized variable from these items coded so that $1=$ yes to any or all of the three drinking variables and 0 =never tried alcohol (see Table 1 for descriptive statistics for all variables).

Table 1. Correlations and Descriptive Statistics for Study Variables $(N=603)$

\begin{tabular}{|c|c|c|c|c|c|c|c|c|c|c|}
\hline Variables & 1 & 2 & 3 & 4 & 5 & 6 & 7 & 8 & 9 & 10 \\
\hline 1. Youth alcohol use & - & & & & & & & & & \\
\hline 2. Youth gender ${ }^{\mathrm{a}}$ & -.03 & - & & & & & & & & \\
\hline 3. Youth age & $.24^{* *}$ & -.05 & - & & & & & & & \\
\hline 4. $\mathrm{FC}^{\mathrm{b}}$ age & $.09^{*}$ & -.02 & $.13^{* *}$ & - & & & & & & \\
\hline 5. Household per capita income ${ }^{\mathrm{c}}$ & $-.10^{*}$ & -.02 & .01 & -.01 & - & & & & & \\
\hline 6. FC binge drinking, past month & $.12^{* *}$ & .01 & .03 & .03 & -.05 & - & & & & \\
\hline 7. FC substance use-related problems & $.14^{* *}$ & .00 & $.10^{*}$ & -.04 & $-.13^{* *}$ & $.21^{* *}$ & - & & & \\
\hline 8. Youth exposure to FC heaviest drinking & .03 & .05 & -.03 & $-.19^{* *}$ & -.06 & $.20 * *$ & $.18^{* *}$ & - & & \\
\hline 9. Coercive parenting & $.11^{* *}$ & $-.10^{*}$ & $-.08^{*}$ & -.05 & .03 & .06 & .00 & -.00 & - & \\
\hline 10. Parental monitoring & $-.16^{* *}$ & .04 & $-.08^{*}$ & $-.14^{*}$ & $.12^{* *}$ & $-.09 *$ & $-.10^{*}$ & $-.15^{* *}$ & -.04 & - \\
\hline$M$ & .17 & .51 & 11.08 & 39.10 & 5.36 & .65 & 2.41 & .19 & 1.39 & 3.24 \\
\hline$S D$ & .38 & .50 & .81 & 9.42 & 3.87 & .82 & 1.95 & .39 & .83 & .82 \\
\hline
\end{tabular}

a. $0=$ male, $1=$ female. b. Female caretaker. c. Per $\$ 1,000$.

${ }^{*} p<.05 ;{ }^{* *} p<.01$ 
Caretaker alcohol use characteristics are measured using three different strategies: heaviest lifetime drinking, alcohol- and drug-related problems, and current binge drinking. Our decision to utilize multiple measures of caretaker alcohol use allows us to better understand how general substance use-related behaviors and attitudes might influence youth differently. First, heaviest lifetime drinking measures the influence of caretaker drinking when it peaks during the youth's late childhood to early adolescence. To create this variable, two separate caretaker questionnaire items were used: (a) What was your age on your last birthday and (b) How old were you during this period of your life when you drank the most? Questionnaire skip patterns were accounted for and nondrinking caretakers coded to 0 as appropriate. The differences between these two responses were dichotomized to over and under 5 years difference. If the caretaker reported that their heaviest period of drinking was within 5 years of the interview, they receive a score of 1 . If this period was more than 5 years prior, their response was coded as a 0 . Therefore, a higher score on this variable is associated with a higher possibility of youth exposure to the caretaker's heaviest drinking. The cutoff of 5 years allows for a target adolescent age of at least 5 years at the adult drinking "peak;" therefore, youth were potentially more aware of their caretaker's alcohol use than at younger ages.

Caretaker alcohol- and drug-related problems are measured by an additive scale that combined adult responses to six questions regarding lifetime drugor alcohol-associated problems, or both. Adults were asked if drinking or drug use interfered with work, home, or school; caused trouble with family and friends; resulted in arrest; required treatment; led to frequent physical fights; and whether they continued to use despite awareness of the problems it caused. Because this measure is indicative of a number of negative consequences associated with substance use, it is meant to capture serious caretaker substance use that may influence youth outcomes more so than nonabusive substance use. Responses $(1=y e s, 0=n o)$ to each of these measures were summed resulting in a variable range of 0-6 where higher values indicate more problems. Cronbach's a for this scale was .78.

Current caretaker binge drinking is measured by adult responses to a question that asks how many drinks (on average) they had in one sitting in the past month. Previous questionnaire items and skip patterns were accounted for by recoding neverdrinking and currently not-drinking adults to a value of 0 . Binge drinking is commonly defined as five or more alcoholic drinks at one time (Naimi et al., 2003). Therefore, the open-ended responses to this question were collapsed into three resulting categories: 0 =not a current drinker, $1=$ current nonbinge drinker (less than five drinks), and 2 =current binge drinker (greater or equal to 5 drinks).

Levels of parental monitoring are measured from youth responses on questions that asked how often in a usual day someone in their family knows where they are, and how often someone knows when they came home or are in by a set time (response categories: 0 =never, 1 =sometimes, and 2 $=$ always). The responses to these two questions were summed, resulting in a variable ranging from 0 to 4 .

Coercive parenting was constructed identically to parental monitoring. Children were asked how often they were (a) yelled at or (b) spanked for misbehaving. The responses to these two questions were summed, resulting in a range between 0 and 4.

Youth Gender is controlled for using a dummy variable where $0=$ male and $1=$ female.

Female caretaker age is controlled for using a continuous measure of open-ended female adult responses to a question assessing how old the person was on their last birthday.

Youth age is constructed identically to female caretaker age but represents youth responses to how old the target adolescent was on the last birthday.

Last, we include a control variable measuring household per capita yearly income. First, families were asked to indicate whether their overall household incomes were above or below \$25,000 in the past year. Two additional questions narrow these responses to within $\$ 10,000$ ranges. The midpoints of each of these ranges were used to sum the two variables, which were then divided by the number of people living within the household at least $50 \%$ of the time, thus assessing per capita family income.

Our analysis of missing values revealed no significant differences between those who completed and those who did not respond to each item. Two of our measures of female caretaker alcohol-related behaviors showed missing responses for $3.5 \%$ of the data, with all other variables missing even fewer or no cases. Despite such limited miss- 
ing data, we chose to maximize the use of full data by imputing missing values via EM algorithm-derived maximum likelihood estimates. Such estimation is advantageous over commonly used techniques for dealing with missing data (i.e., listwise deletion, mean substitution) that may increase biased estimates and lead to invalid conclusions (see, e.g., Acock, 2005; Allison, 2002).

\section{Results}

Table 1 shows descriptive statistics and bivariate correlations for all variables. Although the variables appear to be somewhat skewed, a q-q plot showed a distribution of residuals that approximated multivariate normality (Cohen, Cohen, West, \& Aiken, 2003). Note the levels of income reported by families: Yearly per capita household income ranges from a very low $\$ 250$ to $\$ 25,000$. The mean of $\$ 5,530$ is well below the 2002 annual poverty threshold for one-person households of $\$ 9,183$ (U.S. Census Bureau, 2002).

Over $17 \%$ of the adolescents said that they had at least experimented with alcohol use at the time of their interviews. Among female caretakers, $41.9 \%$ report current drinking. Of these current drinkers, 23\% report binge drinking (not shown). In addition, $19 \%$ of these caretakers reported their heaviest period of drinking as occurring sometime in the past 5 years; thus, a number of children were likely exposed to their caretaker's heavy drinking sometime after their fifth birthdays.

\section{Bivariate analyses}

There are significant bivariate relationships between caretaker and adolescent alcohol use in terms of caretaker reports of drug and alcohol problems and current caretaker binge drinking. These findings provide modest support for a social learning influence on adolescent drinking.

Both parenting measures are also significantly related to adolescent alcohol use at the bivariate level. Coercive parenting is positively associated and parental monitoring negatively associated with early-onset alcohol use. Both of these results support the hypothesized directions for relationships among these variables. In terms of the effects of caretaker alcohol behaviors on parenting skills, only parental monitoring is negatively associated with all three measures of adult substance use; contrary to our expectations, adult alcohol-related behaviors are not associated with co- ercive parenting at the bivariate level. Among the control variables, being an older youth, having an older female caretaker, and living in homes with lower per capita income are all positively associated with early-onset youth alcohol use.

Several more significant bivariate associations between independent variables are worth noting. For example, being a female adolescent is associated with experiencing lower levels of coercive parenting. Youth age is positively associated with coercive parenting and negatively associated with parental monitoring. Being an older female caretaker is positively associated with age of youth and negatively associated with both youth's exposure to the caretaker's heaviest drinking and parental monitoring. Household per capita income is negatively associated with female caretaker reports of drug- and alcoholrelated problems and positively associated with parental monitoring. Each of the female adult substance use behavior measures is positively correlated with one another, but the strength of these associations (none higher than $r=.21$ ) does not suggest an issue with collinearity for our final model.

\section{Multivariate analyses}

A fully recursive path model was tested for all variables (Figure 2). The final model explains $25 \%$ of the variance in youth alcohol use. The significant paths for this model are shown in Figure 2 (see Table 2 for probit coefficients for the full model). Because our model contains both categorical (youth alcohol use) and continuous (parenting items) endogenous variables, the resulting coefficients are probit estimates. Probit analyses allow for estimates of the effects of variables on dichotomous outcomes by assuming a latent continuous measure that underlies the categorical manifest variable.

When controlling for all other variables in the model, the direct effect expected (H1) between adult and youth substance use is supported only for the measure of female caretaker binge drinking. Also as expected $(\mathrm{H} 2)$, caretaker reports of heaviest lifetime drinking occurring within the past 5 years are negatively associated with parental monitoring. In turn, we find support for $\mathrm{H} 3$ in that coercive parenting is positively and parental monitoring negatively associated with the outcome variable of youth alcohol use. Despite our expectations, however, there are no associations 


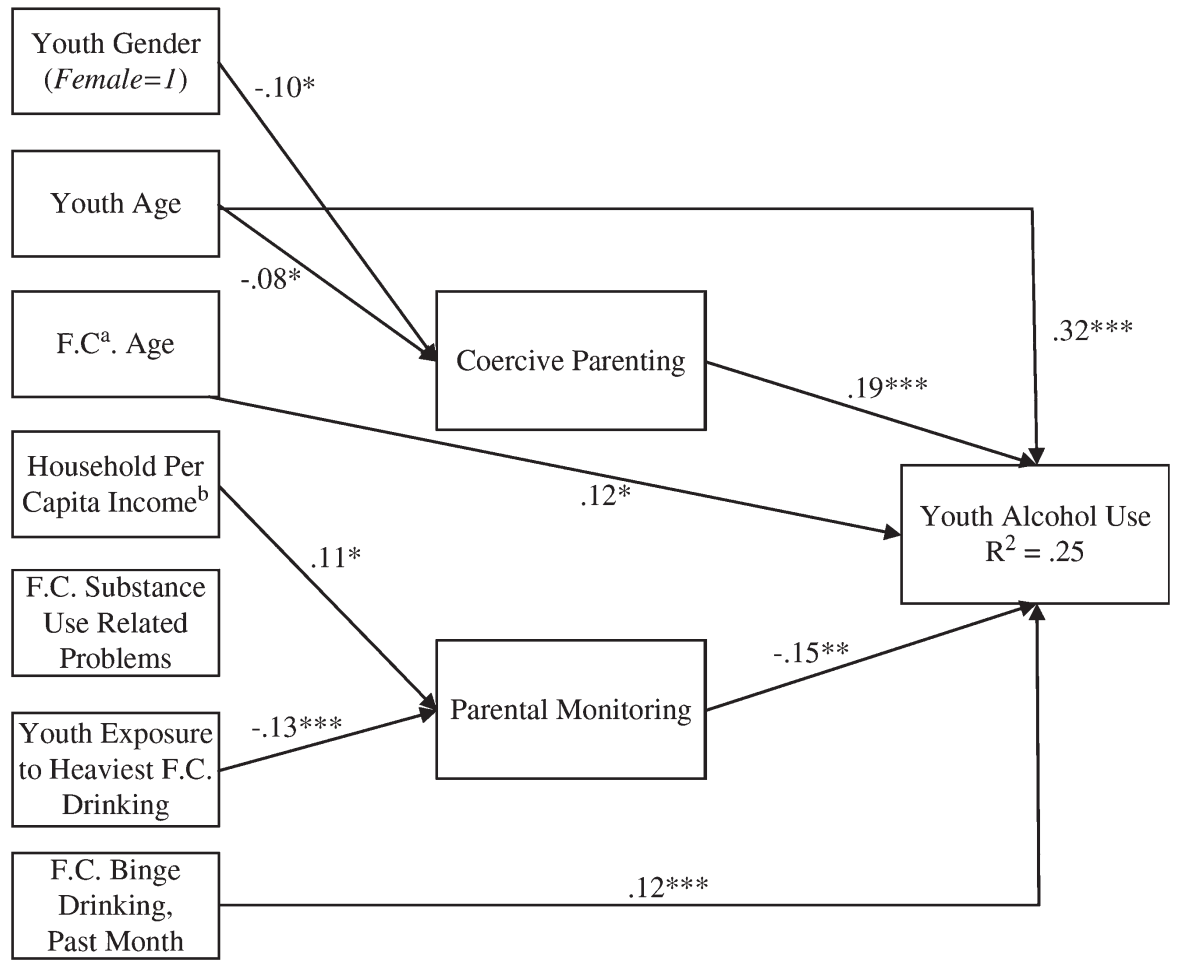

Figure 2. Path Model for Adolescent Alcohol Use. a. F.C. $=$ female caretaker. b. Per $\$ 1,000 .{ }^{*} p<.05 ; * * p<.01$; $* * * p<.001$.

between female caretaker substance use-related problems and parenting behaviors, nor between substance use problems and early-onset youth alcohol use.

Only one of our hypothesized relationships between the control variables and youth alcohol use $(\mathrm{H} 4)$ is supported in the multivariate analy- ses. As expected, older youths are more likely to report early onset alcohol use than their younger peers. The only remaining control variable significantly associated with youth alcohol use in the path model is female caretaker age: Contrary to our expectations, youth living with older female caretakers are more likely to have engaged in early

Table 2. Probit Regression Coefficients and Standard Errors for Variables Regressed on Parenting and Youth Alcohol Use

\begin{tabular}{lccc}
\hline Variable & Coercive Parenting & Parental Monitoring & Youth Alcohol Use \\
\hline Youth gender & $-.10^{*}(.07)$ & $.05(.07)$ & $.001(.13)$ \\
Youth age & $-.08^{*}(.04)$ & $-.08(.04)$ & $.32^{* * *}(.08)$ \\
FC age & $-.04(.00)$ & $-.08(.00)$ & $.12^{*}(.01)$ \\
Household per capita income & $.03(.01)$ & $.11^{*}(.01)$ & $-.13(.02)$ \\
FC binge drinking, past month & $.06(.04)$ & $-.05(.04)$ & $.12^{*}(.08)$ \\
FC substance use-related problems & $.001(.02)$ & $-.05(.02)$ & $.11(.03)$ \\
Youth exposure to FC heaviest drinking & $-.02(.09)$ & $-.13^{* * *}(.08)$ & $.001(.16)$ \\
Coercive parenting & & $.19^{* * *}(.06)$ & \\
Parental monitoring & & $-.15^{* *}(.08)$ & \\
\hline
\end{tabular}

a. $0=$ male, 1 =female. b. Female caretaker. c. Per $\$ 1,000$.

${ }^{*} p<.05 ;{ }^{* *} p<.01 ; * * * p<.001$ 
onset alcohol use. In addition to these tests of hypothesized relationships, being a younger or female youth is negatively associated with experiencing coercive parenting. Those living in homes with higher per capita incomes report experiencing higher rates of monitoring as well.

To illustrate the total direct and indirect associations among the paths tested in this model, we obtained estimates derived from a decomposition of effects. Only two indirect influences reached statistical significance (both are path specific rather than a sum of indirect effects). First, there is a significant indirect effect of youth exposure to the female caretaker's heaviest period of lifetime drinking $(.02, p<.05)$ that occurs via lessened parental monitoring. Second, being female produces a slight negative indirect effect on earlyonset alcohol use $(-.02, p<.05)$ through less coercive parenting.

\section{Discussion}

The purpose of this paper was to examine the effects of caretaker drinking behaviors on earlyonset alcohol use among Native American adolescents. On the basis of previous research (Barnes et al., 1986; Sieving et al., 2000), we proposed and found modest support for our hypothesis (H1) of a social learning effect in which youth begin drinking in part because they have observed such behavior by their adult caretakers or parents. Significant bivariate associations were found between female caretaker drug and alcohol problems and current binge drinking with early-onset youth alcohol use. In our path model, a direct effect was found for adult female caretaker reports of past month binge drinking. These findings are evidence of a direct modeling effect of caretaker substance use on youth substance use outcomes. Binge drinking is our most robust measure of current female caretaker alcohol use behaviors and was the only adult alcohol variable to remain significantly associated with the dependent variable in the multivariate model, suggesting that more proximal alcohol use by adults might increase the likelihood of early youth drinking in terms of a social modeling effect.

Also consistent with past research (DiClemente et al., 2001; Windle, 1996) are the hypothesized (H2) significant associations between caretaker drinking behaviors and less effective parenting processes. Our hypothesis that all the measures of caretaker drinking characteristics would negatively affect parenting was not fully supported. At the bivariate level, all three female caretaker alcohol measures were significantly associated with lower parental monitoring. In the path model, only our assessment of youth exposure to the female caretaker's heaviest period of drinking remained negatively associated with monitoring. It may be that this peak in lifetime drinking sometime within the past 5 years is indicative of a period during which parental monitoring levels were decreased as a result of increasing levels of alcohol consumption.

Contrary to our expectations, we found no significant associations between our measures of female caretaker alcohol behaviors and coercive parenting. This is especially surprising considering comments provided by participants of focus groups conducted as part of the research project. As one elder remembered: "my dad - when he drank - was altogether bad. That alcohol changed him completely you know, from an easygoing person to ... well, we used to call him the devil." Another elder agreed, "when there was alcohol involved, there was anger." Yet another remembered how alcohol affected the entire family: " ... when my mother knew he (my father) was drinking we had to go and hide somewhere ... even if we had to go in the brush." These comments illustrate the relationship between periods of heavy drinking and negative, coercive parenting styles; in the two instances when caretaker gender was mentioned in these quotations, however, both were men/fathers. It may be that coercive parenting is a gendered process within this particular cultural context, both for parents and for youth. As noted, our analyses also revealed significant youth gender effects on coercive parenting in that female youth were less likely to report experiencing coercion than their male counterparts. Future research should examine these effects by oppositeand same-gender parent and across a variety of Native American cultural groups.

Also, contrary to our hypothesis (H4), female caretaker age was positively associated with earlyonset youth alcohol use at both the bivariate and the multivariate levels. Although there is a negative bivariate association between female caretaker age and parental monitoring, there is no significant relationship in the multivariate model that would indicate that the effects of female caretaker age are indirect via the parenting variables. It may be that older caregivers monitor less and these ef- 
fects are lost in the multivariate analysis; it is also possible, however, that the older female caretakers are actually grandmothers or aunties to the target adolescents in our study. This finding could indicate that the women from older generations serve as caretakers in a vacuum created by the absence or disengagement of the children's younger mothers. If this is true, rather than a generational effect (i.e., the elder female caregivers are more permissive or less effective as caregivers), the association could be spurious reflecting instead the effects of mother absence or disengagement.

We did find support for our hypotheses that youth age and household per capita income would be negatively associated with youth early-onset alcohol use. For youth age, this association is supported in both our bivariate and path analyses and is consistent with prior research (Wallace et al., 2003). Although the negative relationship between household income and youth alcohol use is significant only at the bivariate level, this finding supports prior work (Hawkins et al., 1992), suggesting that income acts as a protective factor against adolescent drinking only in high-poverty situations. Because most of the families in our sample are living at or below poverty levels, our reports of the relationships between income and the remaining variables should be interpreted as results yielded within a low-income group.

The findings presented here concerning the prevalence of alcohol use for both adolescents and their caretakers help to break down the widely shared stereotype that associates all Native Americans with alcoholism. A large majority of adolescents in this sample have never tried alcohol, and almost three quarters of their female caretakers either do not currently use alcohol or drink only moderate amounts. Although this is not meant to undermine the reality of problems surrounding alcohol and substance use on reservations, it demonstrates that many Native Americans in our sample are abstaining from substance use.

In summary, these findings extend research with non-Native families that links nonoptimal parenting to early-onset alcohol use (Barnes et al., 2000; Beck et al., 2003) to Native American families. Consistent with coercion theory, coercive parenting was positively related to youth alcohol use and parental monitoring was negatively associated with youth alcohol use. There was also evidence of direct modeling of drinking behaviors.
Social learning and coercion theory both operate to some degree to explain the risk of early-onset adolescent alcohol use among Native American youth. This research supplements prior work (e.g., Reid et al., 2002) that emphasizes family-based approaches to prevention and intervention programs. Mail and Heurtin-Roberts (2002) point out that studies such as this one are especially important in terms of informing policy, programming, and theory for Native American groups:

Where there is promising non-Indian based research addressing preventive interventions and treatment approaches, this information seems rarely to be adapted or applied to Indian AOD (alcohol or drug) abuse strategies. A commonly given reason for such oversight is that such research is not culturally appropriate because it was "not done with Indian people." (p. 459)

As illustrated in the preceding quotation, the cultural specificity of this work lends substantial credibility to the inclusion of caretaker and parenting variables in Native American youth substance use research. Although not necessarily exceptional, the $R^{2}$ reported for this research $(25 \%)$ is considerable in that the model relies mainly on caretaker-related characteristics as correlates of early alcohol use among youth as opposed to other sources of influence (e.g., peers).

\section{Limitations and future research}

There are several limitations to this research, beginning with the exclusion of male caretaker reports from these analyses. Future work will need to include the influence of fathers on early-onset alcohol use among their children. It may be that caretaker gender plays a prominent role in how adults influence young boys and girls, especially in consideration of gendered behaviors and practices in many traditional Native American cultures. Also, the cross-sectional design of this study does not allow for an inference of causality among the supported hypotheses in this research. For example, those youth who use alcohol may also be more likely to report coercive parenting strategies. Or, it may be that delinquent behaviors such as alcohol use prompt more coercive parenting strategies. Replication of this design using subsequent waves of data would add considerable support to the conclusions presented here.

Another important limitation is our use of only children's reports of parent or caretaker monitor- 
ing and coercive parenting. Consideration of the cultural emphasis on youth respect for adults and elders in this sample could indicate that youth might be reluctant to be critical of their adult caregivers. At the same time, however, the pressures of social desirability associated with being a good parent makes parents or caregivers notoriously poor reporters of monitoring and coercive parenting. This may be particularly true when there has been a history of alcohol abuse. Simons, Johnson, and Conger (1994) have argued that the potential negative effects of coercive parenting are moderated by children's perceptions of parental support. We opted to use only child reports in that we believed that their perceptions of coercive parenting and monitoring would be more accurate than those of parents or caretakers. Another measurement limitation is based on extended family influences within this culture. The traditional extended family system implies there may be multiple intergenerational influences beyond just the parent or caregiver. Although our measures of "parenting" are purposely vague (i.e., "how often in a usual day does someone in your family. ..") to facilitate adolescents' conceptualization of caretaking behaviors from multiple sources, this vagueness introduces new measurement weaknesses in that it is unclear in these analyses who the exact person of reference might be. Given these measurement limitations, the strengths of the associations between parent or caregiver alcohol abuse and child outcomes are particularly significant. In addition, although the sample adequately reflects the variability within one large Native culture, it only includes data from one specific Native American culture in the upper Midwest, thus limiting the ability to generalize these findings across all Native American groups.

A final and very important limitation of this work is our emphasis on contemporary Native family context without due investigation of the lasting effects of historical traumas on Native communities. A history of forced separation of Native American children and their families via attendance at government-run boarding schools in both the United States and Canada has had lasting effects on the Native family (i.e., Duran \& Duran, 1995; Kirmayer et al., 2000). Such coercive separation meant that these children missed out on pivotal cultural socialization practices-including witness to models of positive parenting - through- out their development. Although these children are not in our sample, many of their parents and grandparents endured this wounding historical era. The intergenerational effects of growing up in boarding schools represents an important part of contemporary Native American life that has been connected to mental distress (Brave Heart, 1998, 1999a, 1999b; Brave Heart \& DeBruyn, 1988), including guilt, hopelessness, despair, anger, and depression (Whitbeck, Adams, Hoyt, \& Chen, 2004), and deserves empirical investigation in future works on Native families.

Despite these limitations, this study addresses a serious gap in research: The lack of understanding of parent or caretaker effects on young Native American substance use (Beauvais, Jumper-Thurman, \& Plested, 2002). Parent or caretaker alcohol misuse can increase the risk of early-onset drinking for their children both through modeling and by decreasing effective parenting; there is much to be done, however, to increase our understanding of Native American family influences on youth alcohol use. Future research should address the roles of modeling and direct socialization by relatives other than parents or caretakers, particularly grandparents, uncles, and aunts in accordance with specific cultural kinship norms. Research should examine multiple "caregivers" insofar as different native family members may perform specific caregiver functions in accordance with traditional cultural ways. Adult influence also may be strongly gendered in some Native American cultures suggesting that boys and girls may listen and attend to behaviors and stories of same more than opposite-gender relatives and model their behaviors. Petraitis, Flay, and Miler (1995) point out that "simply hearing influential role models speak favorably about experimental substance use (ESU) and people who use substances might promote the onset of ESU" (p. 71). Such stories, though meant to be harmless, may in fact reinforce perceptions of substance use as a positive experience for children if and when caretakers recount experiences through enthusiastic and often humorous storytelling. Caregiver experiences may be particularly important given the importance and reverence for elders within this culture. Possible caretaker reminiscing along with the high positive regard and respect for elders may actually heighten the possibility that drinking behaviors are transmitted from older family members to young adolescents (Bandura, 1977). 
Although this research indicates that parent or caretaker influence within this Native American culture acts similarly to that in nonnative cultures, there are still cultural nuances of generational effects, kinship patterns, and gendered family roles that need to be explored if we are to more fully understand the cultural variations of family influence across Native American communities. Future research should work to clarify areas where family processes are similar to majority culture and those that are culturally specific.

Acknowledgments - This study was funded by grants from the National Institute on Drug Abuse (DA 13580) and the National Institute of Mental Health (MH67281), L. B. Whitbeck, principal investigator. The authors gratefully acknowledge comments received on drafts of the manuscript from members of this project's community-based advisory boards and from anonymous reviewers.

\section{References}

Acock, A. C. (2005). Working with missing values. Journal of Marriage and Family, 67, 1012-1028.

Akers, R. L., Krohn, M. D., Lanza-Kaduce, L., \& Radusevich, M. (1979). Social learning and deviant behavior: A specific test of a general theory. American Sociological Review, 44, 636-655.

Allison, P. (2002). Missing data. Thousand Oaks, CA: Sage.

Anderson, A. R., \& Henry, C. S. (1994). Family system characteristics and parental behaviors as predictors of adolescent substance use. Adolescence, 29, 405-420.

Ary, D. V., Tildesley, E., Hops, H., \& Andrews, J. (1993). The influence of parent, sibling, and peer modeling and attitudes on adolescent use of alcohol. International Journal of the Addictions, 28, 853-880.

Bachman, J., Wallace, J., O'Malley, P., Johnson, L., Kurth, C., \& Neighbors, H. (1991). Racial/ethnic differences in smoking, drinking, and illicit drug use among American high school seniors, 19761989. American Journal of Public Health, 81, 372-377.

Bandura, A. (1977). Social learning theory. Englewood Cliffs, NJ: Prentice-Hall.

Barnes, G., Reifman, A., Farrell, M., \& Dintcheff, B. (2000). The effects of parenting on the develop- ment of adolescent alcohol misuse: A six-wave latent growth model. Journal of Marriage and the Family, 62, 175-187.

Barnes, G., \& Farrell, M. (1992). Parental support and control as predictors of adolescent drinking, delinquency, and related problem behaviors. Journal of Marriage and the Family, 54, 763-776.

Barnes, G., Farrell, M., \& Cairns, A. (1986). Parental socialization factors and adolescent drinking behaviors. Journal of Marriage and the Family, 48, 27-36.

Beauvais, F. (1992). The consequences of drug and alcohol use for Indian youth. American Indian and Alaska Native Mental Health Research: Journal of the National Center, 5, 32-37.

Beauvais, F. (1996). Trends in drug use among American Indian students and dropouts, 1975 to 1994. American Journal of Public Health, 86, 1594-1598.

Beauvais, F. (1998). American Indians and alcohol. Alcohol Health \& Research World, 22, 253-259.

Beauvais, F., Jumper-Thurman, P., \& Plested, B. (2002). Prevention of alcohol and other drug abuse among Indian adolescents: An examination of current assumptions. In P. D. Mail, S. Heurtin- Roberts, S. E. Martin, \& J. Howard (eds.), Alcohol use among American Indian and Alaska Natives: Multiple perspectives on a complex problem (pp. 187-209). NIAAA Research Monograph No. 37. Bethesda, MD: U.S. Department of Health and Human Services.

Beck, K. H., Boyle, J. R., \& Boekeloo, B. O. (2003). Parental monitoring and adolescent risk in a clinic population. American Journal of Health Behavior, 27, 108-116.

Brave Heart, M. (1998). The return to the sacred path: Healing the historical trauma and historical unresolved grief response among the Lakota through a psychoeducational group intervention. South College Studies in Social Work, 68, 287-305.

Brave Heart, M. (1999a). Gender differences in the historical grief response among the Lakota. Journal of Health and Social Policy, 10, 1-21.

Brave Heart, M. (1999b). Oyate Ptayela: Rebuilding the Lakota Nation through addressing historical trauma among Lakota parents. Journal of Human Behavior in the Social Environment, 2, 109-126.

Brave Heart, M., \& DeBruyn, L. (1998). The American Indian holocaust: Healing historical unresolved grief. American Indian and Alaska Native Mental Health Research, 8, 60-82. 
Centers for Disease Control and Prevention. (2001). Health guidelines to prevent unintentional injuries and violence. $M M W R, 50$, No. RR-22, 4-14.

Chassin, L., \& DeLucia, C. (1996). Drinking during adolescence. Alcohol Heath \& Research World, 20, 175 Centers for Disease Control and Prevention. (2001). 181.

Cohen, J., Cohen, P., West, S. G., \& Aiken, L. S. (2003). Applied multiple regression/correlation analysis for the behavioral sciences (3rd ed.). Mahwah, NJ: Erlbaum.

DiClemente, R., Wingood, G. M., Crosby, R., Sionean, C., Cobb, B. K., Harrington, K., Davies, S., Hook, E. W., \& Oh, M. K. (2001). Parental monitoring: Association with adolescents' risk behaviors. Pediatrics, 107, 1363-1368.

Donnermeyer, J. (1992). The use of alcohol, marijuana, and hard drugs by rural adolescents: A review of recent research. Drugs \& Society, 7, 31-75.

Duran, E., \& Duran, B. (1995). Native American postcolonial psychology. New York: State University of New York Press.

Ellickson, P., Tucker, J., \& Klein, D. (2003). Ten-year prospective study of public health problems associated with early drinking. Pediatrics, 111, 949-955.

Gfellner, B. (1994). A matched-group comparison of drug use and problem behavior among Canadian Indian and White adolescents. Journal of Early Adolescence, 14, 24-48.

Hardy, J. B., Shapiro, S., Astone, N., Miller, T., Brooks-Gunn, J., \& Hilton, S. (1997). Adolescent childbearing revisited: The age of inner-city mothers at delivery is a determinant of their children's self-sufficiency at age 27 to 33. Pediatrics, 100, 802-809.

Hawkins, E. H., \& Blume, A. W. (2002). Loss of sacredness: Historical context of health policies for indigenous people in the United States. In P. D. Mail, S. Heurtin-Roberts, S. E. Martin, \& J. Howard (eds.), Alcohol use among American Indian and Alaska Natives: Multiple perspectives on a complex problem (pp. 25-48). NIAAA Research Monograph No. 37. Bethesda, MD: U.S. Department of Health and Human Services.

Herring, R. (1994). Substance use among Native American Indian youth: A selected review of causality. Journal of Counseling and Development, 72, 578-584.

Indian Health Service. (1998-1999). Indian health focus: Youth. Rockville, MD: U.S. Department of Health and Human Services.
Jacob, T., \& Johnson, S. L. (1999). Family influences on alcohol and substance abuse. In P. Ott, R. T. Ammerman, \& R. E. Tarter (eds.), Sourcebook on substance abuse: Etiology, epidemiology, assessment, and treatment (pp. 166-174). Needham Heights, MA: Allyn \& Bacon.

Jessor, R. (1987). Problem-behavior theory, psychosocial development, and adolescent problem drinking. British Journal of Addiction, 82, 331-342.

Johnson, P., \& Johnson, H. (1999). Cultural and familial influences that maintain the negative meaning of alcohol. Journal of Studies on Alcohol, 13, 79-83.

Kandel, D. B., Yamaguchi, K., \& Chen, K. (1992). Stages of progression in drug involvement from adolescence to adulthood: Further evidence for the gateway theory. Journal of Studies on Alcohol, 53, 447-457.

Kaplow, J., Curran, P., Dodge, K., \& The Conduct Problems Prevention Research Group. (2002). Child, parent, and peer predictors of early-onset substance use: A multisite longitudinal study. Journal of Abnormal Child Psychology, 30, 199-216.

Kirmayer, L. J., Brass, G. M., \& Tait, C. L. (2000). The mental health of Aboriginal peoples: Transformations of identity and community. Canadian Journal of Psychiatry, 45, 607-616.

Li, C., Pentz, M. A., \& Chih-Ping, C. (2002). Parental substance use as a modifier of adolescent substance use risk. Addiction, 97, 1537-1550.

Mail, P. D., \& Heurtin-Roberts, S. (2002). Where do we go from here? Unmet research needs in American Indian alcohol use. In P. D. Mail, S. HeurtinRoberts, S. E. Martin, \& J. Howard (eds.), Alcohol use among American Indian and Alaska Natives: Multiple perspectives on a complex problem (pp. 459-786). NIAAA Research Monograph No. 37. Bethesda, MD: U.S. Department of Health and Human Services.

May, P. A. (1995). The epidemiology of alcohol abuse among American Indians: The Mythical and real properties. IHS Primary Care Provider, 20, 37-56.

May, P. A. (1982). Substance use and American Indians: Prevalence and susceptibility. International Journal of the Addictions, 17, 1185-1209.

May, P. A. (1986). Alcohol and drug misuse prevention programs for American Indians: Needs and opportunities. Journal of Studies on Alcohol, 47, 187-195.

McGue, M., Iacono, W., Legrand, L., Malone, S., \& Elkins, I. (2001). Origins and consequences of age at 
first drink: I. Associations with substance-use disorders, disinhibitory behavior and psychopathology, and P3 amplitude. Alcoholism: Clinical and Experimental Research, 25, 1156-1165.

Naimi T., Brewer, B., Mokdad, A., Serdula, M., Denny, C., \&. Marks, J. (2003). Binge drinking among U.S. adults. Journal of the American Medical Association, 289, 70-75.

Petraitis, J., Flay, B. R., \& Miler, T. R. (1995). Reviewing theories of adolescent substance use: Organizing pieces of the puzzle. Psychological Bulletin, 117, 67-86.

Plunkett, M. (2000). Substance use rates among American Indian Adolescents: Regional comparisons with monitoring the future high school seniors. Journal of Drug Issues, 30, 575-592.

Reid, J. B., Patterson, G. R., \& Snyder, J. (2002). Antisocial behavior in children and adolescents: A developmental analysis and model for intervention. Washington, DC: American Psychological Association.

Rivers, P. C. (1994). Alcohol and human behavior: Theory, research, and practice. Englewood Cliffs, NJ: Prentice Hall.

Rodgers, K. B., \& Fleming, W. M. (2003). Individual, family, and community factors related to alcohol use among Native American adolescents. Adolescent and Family Health, 3, 140-147.

Sieving, R., Maruyama, G., Carolyn, L., Williams, C. L., \& Perry, C. (2000). Pathways to adolescent alcohol use: Potential mechanisms of parent influence. Journal of Research on Adolescence, 10, 489-514.

Simons, R. L., Johnson, C., \& Conger, R. D. (1994). Harsh corporal punishment versus quality of parental involvement as an explanation of adolescent maladjustment. Journal of Marriage and the Family, 55, 713-723.
Substance Abuse and Mental Health Services Administration. 2002. National survey on drug use and health. SAMHSA, Office of Applied Studies.

Swaim, R. C., Oetting, E. R., Thurman, P. J., Beauvais, F., \& Edwards, R. W. (1993). American Indian adolescent drug use and socialization characteristics. Journal of Cross-Cultural Psychology, 24, 53-70.

U.S. Census Bureau. (2002). Poverty thresholds for 2002 by size of family and number of related children under 18 years. Washington, DC: Author. Retrieved July 9, 2004, from http://www.census.-gov/hhes/ poverty/threshld/thresh02.html

Wallace, J. M, Jr., Bachman, J. G., O'Malley, P., Schulenberg, J., Cooper, S., \& Johnston, L. (2003). Gender and ethnic differences in smoking, drinking, and illicit drug use among American 8th, 10th, and 12th grade students, 1976-2000. Addiction, 98, 225-234.

Whitbeck, L. B., Adams, G. A., Hoyt, D. R., \& Chen, X. (2004). Conceptualizing and measuring historical trauma among American Indian people. American Journal of Community Psychology, 33, 119-130.

Wills, T., \& Yaeger, A. (2003). Family factors and adolescent substance use: Models and mechanisms. Current Directions in Psychological Science, 12, 222-227.

Windle, M. (1996). Effect of parental drinking on adolescents. Alcohol Health \& Research World, 20, 181-185. 